\title{
Optimizing Opponents Selection in Bilateral Contracts Negotiation with Particle Swarm
}

\author{
Francisco Silva ${ }^{1}$, Ricardo Faia ${ }^{1}$, Tiago Pinto ${ }^{12}$, Isabel Praça ${ }^{1}$, and Zita Vale ${ }^{1}$ \\ 1 GECAD - Knowledge Engineering and Decision Support Research Center, Institute \\ of Engineering - Politechnic of Porto (ISEP/IPP), Porto, Portugal * \\ 2 BISITE - Research Centre, University of Salamanca, Salamanca, Spain \\ \{f spsa,rfmfa, tmcfp, icp, zav\}@isep.ipp.pt \\ ${ }^{2}$ tpinto@usal.es
}

\begin{abstract}
This paper proposes a model based on particle swarm optimization to aid electricity markets players in the selection of the best player(s) to trade with, to maximize their bilateral contracts outcome. This approach is integrated in a Decision Support System (DSS) for the pre-negotiation of bilateral contracts, which provides a missing feature in the state-of-art, the possible opponents analysis. The DSS determines the best action of all the actions that the supported player can take, by applying a game theory approach. However, the analysis of all actions can easily become very time-consuming in large negotiation scenarios. The proposed approach aims to provide the DSS with an alternative method with the capability of reducing the execution time while keeping the results quality as much as possible. Both approaches are tested in a realistic case study where the supported player could take almost half a million different actions. The results show that the proposed methodology is able to provide optimal and near-optimal solutions with an huge execution time reduction.
\end{abstract}

Keywords: Automated Negotiation, Bilateral Contracts, Decision Support System, Electricity Markets, Game Theory, Particle Swarm Optimization

\section{Introduction}

Over the last decades, the Electricity Markets (EMs) suffered profound changes to adapt themselves to the society's needs and the arising challenges. The current EMs models are a result of the previous privatization, liberalization and the international integration of national systems [1]. These changes have been

\footnotetext{
* This work has received funding from the European Union's Horizon 2020 research and innovation programme under the Marie Sklodowska-Curie grant agreement No 641794 (project DREAM-GO) and grant agreement No 703689 (project ADAPT); from the CONTEST project - SAICT-POL/23575/2016; and from FEDER Funds through COMPETE program and from National Funds through FCT under the project UID/EEA/00760/2013.
} 
motivated by the increased use of energy from renewable sources. An example of the current trend is the long-term plan of the European Union (EU) to reduce greenhouse gas emissions by $80 \%$ in 2050 [2]. The EU targeted the increase of the renewable sources share in the EU total consumption to at least $20 \%$ in 2020 and $27 \%$ in 2030. The latest results show that EU is on course to meet its objective as the share was doubled from $8.5 \%$ in 2004 to $17 \%$ in 2016 .

While the evolution of the sector enriched its models, it also made them more complex, unpredictable and difficult to follow by the involved entities. Therefore, these entities need proper tools that allow them to have a better insight of the EMs operation. The EMs simulation has been proving to be a good solution as the entities are able to study the markets mechanisms and the players' relations, exploring their strategies. However, the current simulators are mostly focused in the auction-based market models, not exploring the bilateral contracts model. A relevant review [3] identifies the main phases of automated negotiation: prenegotiation, actual negotiation and renegotiation. As result of their study, the authors identified the actual negotiation as the most explored phase while the pre-negotiation lacks further exploration, specially regarding opponents analysis.

In the literature, is possible to find some tools that support bilateral contracts negotiation such as EMCAS [4], GENIUS [5] and MAN-REM [6]. EMCAS is a multi-agent simulator that is able to simulate EMs bilateral contracts, established between a demand agent and a generation company agent. The generation agents decide the price of the demand agents' proposals that may or may not be accepted by the proposers. GENIUS is a multi-agent simulator that facilitates and evaluates the strategies of automated negotiators. The tool supports domain-independent bilateral negotiations and considers three negotiation phases: Preparation (negotiation protocol and domain), Negotiation, and Postnegotiation (negotiation analysis). MAN-REM simulates the bilateral contracts negotiation through the combination of small multi-agent simulators. The tool models the buyer, seller, distributor, and market operator (validation) agents. Three negotiation phases are considered: Pre-Negotiation (contract's preferences and response to counter-offers definition), Actual Negotiation, and PostNegotiation (final agreement). The analysed tools present a lack of exploration of the pre-negotiation, focusing the actual negotiation. The GENIUS simulator has the most explored pre-negotiation but also lacks an opponents analysis.

The identified lack of proper opponent analysis, in the pre-negotiation phase, motivated the development of a new Decision Support System (DSS) [7]. The tool is capable to analyse the possible opponents that the supported player may face and identify the one(s) that may guarantee the best negotiation outcome. For this purpose, the DSS uses a game theory based approach, which selects the best of all the possible actions that the supported player can take, under several different scenarios. Although this approach always finds the optimal solution, its execution time greatly increases as the amount of energy to trade and the number of possible opponents increases. Thus, this paper proposes an alternative approach by using Particle Swarm Optimization (PSO) [8], a meta-heuristic algorithm, to reduce the time required to obtain a good solution. 


\section{Decision Support System for the Pre-Negotiation of Bilateral Contracts}

The developed DSS for the pre-negotiation of bilateral contracts [7] aims to aid the supported player through the process of identifying the best opponents to trade with, and how much to trade with each one, to maximize the negotiation outcome, considering its objectives. The supported player may want to maximize its profit or cost, depending if it is selling or buying, but also consider other aspects such the opponents reputation, worst scenario, most probable scenario and optimal scenario.

To reach this objective, the DSS has three phases: (i) scenarios definition, (ii) possible actions, and (iii) decision process. Firstly, the scenarios definition is feeded with data by a database with historical bilateral contracts established by other players. By accessing this information, the DSS generates a set of possible scenarios that the supported player may face. Each scenario is a set of the expected prices that each possible opponent may offer for all the energy amounts between 1 and the total amount to trade.

On the second phase, following a game theory approach, the DSS generates all the possible actions (total energy's distributions among the possible opponents) that the supported player can take under each scenario [9].

After the possible actions generation, on the third and last phase, there is the need to assess the utility of each action to be able to identify the best one, regarding the objectives of the supported player. For this purpose, the utility of each action results from the weighted sum of two components: economic and reputational. The economic component quantifies how economically advantageous is an action while the reputational component measures the average reputation of the involved opponents. The weight of each component depends on the percentage of risk that the supported player is willing to face. The higher the risk the higher the weight of the economical component. The minimum risk (0\%) means that only the reputational component is considered, ignoring the economic component, while maximum risk has the opposite meaning. The utility assessment is performed for every action of every scenario.

The decision process ends with the selection of the best action, which depends on the selected decision method by the supported player. For this purpose, the DSS offers three methods: Optimistic, Most Probable and Pessimistic. The Optimistic method selects the action with the highest utility value among all the scenarios. The Most Probable method selects the action with the highest utility value of the most probable scenario, the scenario that is the most likely to occur in reality. The most probable scenario is determined by using a q-learning approach which keeps evaluating the realism of the generated scenarios every time the tool's database is feeded with the real negotiation scenarios. At last, the Pessimistic decision method, is based on the mini-max game theoretic approach, by selecting the action with the highest utility of the scenario with the lowest global utility. The global utility of a scenario is the sum of the actions' utility when taken in that scenario [9]. 


\section{Proposed Methodology}

This paper proposes the use of PSO, a meta-heuristic algorithm, to determine the best action the supported player can take, without the heavy task of analysing all the possible actions.

Introduced by Kennedy and Eberhart in 1995 [8], the PSO algorithm was inspired by the movement of organisms in large swarms such as bird flocks or fish schools. The algorithm optimizes a problem by starting with an initial swarm of particles, randomly positioned in the search-space, and then moving them towards the best solution by improving their best positions over time. As result, the method is able to present good solutions but does not guarantee the optimal solution. The algorithm applies the equations 1 and 2 at each iteration.

$$
v_{i d}^{k+1}=w \cdot v_{i d}^{k}+c_{1} \cdot r_{1}^{k} \cdot\left(\text { Pbest }_{i d}^{k}-x_{i d}^{k}\right)+c_{2} \cdot r_{2}^{k} \cdot\left(G b e s t_{i d}^{k}-x_{i d}^{k}\right)
$$

$$
x_{i d}^{k+1}=x_{i d}^{k}+v_{i d}^{k+1}
$$

Where,

- Pbest - best position found by each particle,

- Gbest - best position of all particles,

$-v$ - velocity of the particle,

$-x$ - position of the particle,

$-k$ - iteration,

$-d$ - parameter,

$-i$ - particle,

- $w$ - inertia term,

- $c_{1}$ - local attraction term,

- $c_{2}$ - global attraction term,

- $r 1, r 2$ - random number between $[0,1]$.

The algorithm 1 presents the main process of the PSO.

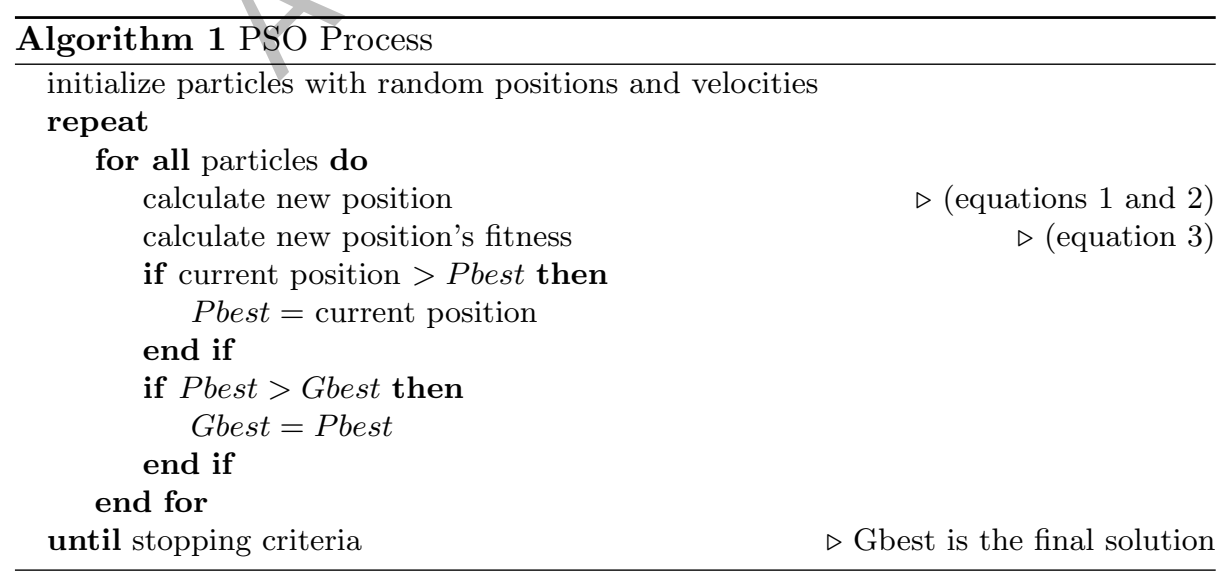


As specified in the algorithm 1, the PSO's process begins with the assignment of a random position and velocity to each particle. At each iteration, their position is updated, by applying equations 1 and 2, and evaluated by the objective function (equation 3). Then, if a better position is found, it becomes the local best and also global best if it is higher than the current value. When reached the stopping criteria, the process ends with the global best position as the final solution.

The process of the PSO method depends on the following parameters:

- Swarm size - the number of particles of the swarm. The bigger the swarm size, the bigger the covered area of the search space, in each iteration; The large swarms reduce the number of iterations but increase their complexity and, consequently, the execution time to obtain a good solution.

- Maximum number of iterations. This parameter must be adapted to each problem as the best value is the minimum one which guarantees a good solution. The value can be too low if it ends the search without finding a good solution and too high if only increases the execution time without improving the final solution.

- Local and global attraction terms - the confidence that a particle has on the previous searches of itself and its neighbours, respectively.

- Inertia weight - a limit to the particles movement which controls how much they can move in a single iteration.

The equation 3 presents the formulation of the optimization problem.

$$
f= \begin{cases}\min \left(\sum_{i=1}^{N i}\left(A_{i} \times E P_{i, A}\right)\right), & \text { if negotiation type }=1 \\ \max \left(\sum_{i=1}^{N i}\left(A_{i} \times E P_{i, A}\right)\right), & \text { if negotiation type }=2\end{cases}
$$

Where,

$-i$ - player,

- Ni - number of players,

- $A_{i}$ - amount of energy to trade with player $i$,

- $E P_{i, A}$ - expected price of player $P$ for the energy amount $A$,

- Negotiation Type - 1 when the supported player is buying and 2 when selling.

The expected prices result from the forecasts performed by the DSS and, for the energy amounts that were not possible to forecast, an estimation process is performed. The estimation process combines a clustering mechanism with a fuzzy approach [10].

The main constraint applied to this problem is represented by equation 4 . It guarantees that the sum of the amount to trade with each player is exactly the total amount $T A$ that the supported player wants to trade.

$$
\sum_{i=1}^{N i}\left(A_{i}\right)=T A
$$




\section{Experimental Findings}

This section presents a case study to validate the proposed methodology and compare it with the game theory approach presented in section 2. For this purpose, both approaches are used to solve the same scenario and their results are compared.

In this case study scenario, the supported player wants to sell $55 \mathrm{MWh}$, in a weekday context, and indicates five possible opponents that it may trade with. The supported player does not want to consider the negotiation risk, ignoring the opponents' reputation, and chooses the Most Probable decision method. In this scenario, the supported player can take 455126 different actions. The game theory approach will evaluate each of these actions to select the most profitable one while the PSO approach will attempt the same without testing all actions.

This case study is based on real data by using real executed physical bilateral contracts declared in the Iberian Market Operator [11]. However, the dataset does not contain the contracts' established price as it is a key information and therefore it is rarely shared. The missing prices werê generated by considering five different price profiles based on the real Iberian Day-ahead market price [11]. The profile 1 ranges from $70 \%$ to $130 \%$ of the market price, profile 2 is an increased version of profile 1 by $5 \%$, profile 3 follows the market price, and profiles 4 and 5 have the opposite trend of profiles 2 and 1, respectively. The possible opponents are Player 1 (Profile 1), Player 2 (Profile 2), Player 3 (Profile 3), Player 4 (Profile 4) and Player 5 (Profile 5), which represent real players of the Iberian Market. The selected players are the most active ones in the dataset, whom frequently establishes contracts with a lower or equal energy amount than the considered for this scenario.

The PSO algorithm is executed 100 times with the following parameters: 10 particles, 150 iterations, $[0.4,0.9]$ inertia interval, and 1 local and global attraction terms. The DSS's price forecasts combined with the estimation methodology produced the expected prices presented in Figure 1.

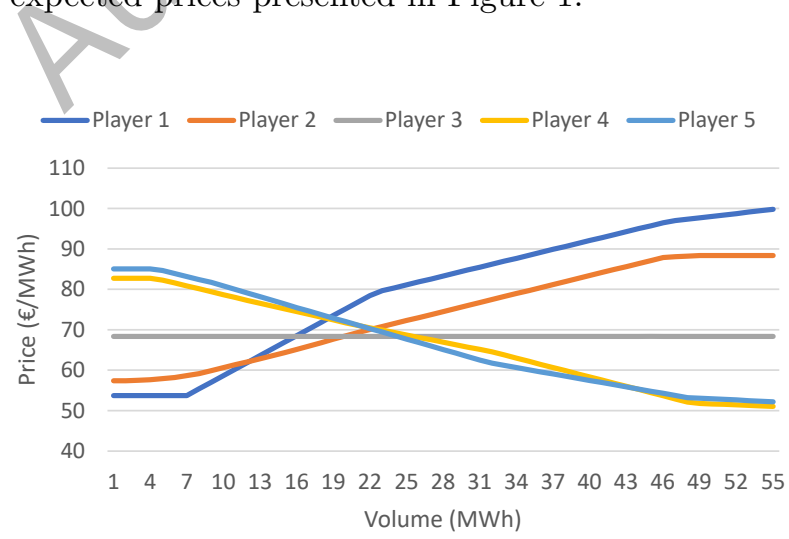

Fig. 1: Expected prices for each possible opponent 
As it can be observed in Figure 1, the expected prices for each opponent, over the different energy amounts, follow the trend of their price profile. In this scenario, it is clear that the best deal for the supported player is to sell all the energy to Player 1, which presents the highest price for $55 \mathrm{MWh}$. This was proven by the game theory approach which identified this trade as the most profitable, after an execution of 58.68 minutes. On the other hand, the PSO approach was able to obtain the same result, finding the optimal solution without a significant cost of time ( 14.07 seconds, $4 \%$ of the game theory approach's execution time). The Figure 2 and Figure 3 show the difference of the execution time of both approaches for the same scenario but different energy amounts.

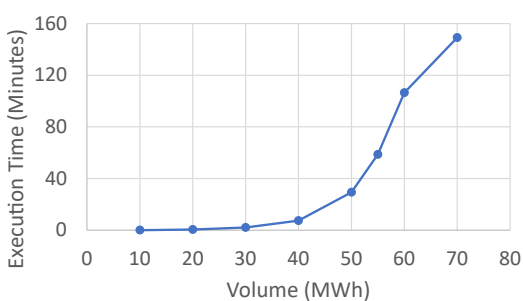

Fig. 2: Execution time of the Game Theory approach

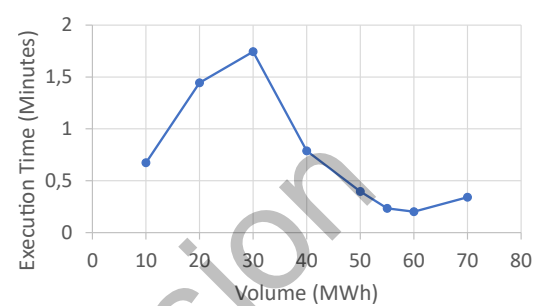

Fig. 3: Execution time of the PSO approach

The Figures 2 and 3 evidence the great execution time difference of both approaches. While the first approach can take up to 150 minutes to present a solution, for the biggest scenario observed, the PSO approach takes less than 2 minutes in its longest execution observed. The execution time of the game theory approach depends on the energy amount to negotiate. Even a small increase of the amount of energy to negotiate can greatly increase its execution time as the number of possible actions greatly increases. In this scenario, the supported player can take 455126 different actions but, if the energy to negotiate is increased by 5 , the number of different actions is increased by $39.6 \%$ (635376). Regarding the PSO approach, the trend is completely different. The highest impact to the execution time is not the amount of energy but the expected prices for that amount. As it can be seen in Figure 3, the method has longer execution times for scenarios with an higher number of good solutions, which is the case of the scenarios with an energy amount between $10 \mathrm{MWh}$ and $40 \mathrm{MWh}$. On the remaining cases, the optimal solution is easier to be found, and thus quicker.

The recommended trade by both approaches results in a profit of 5489.55 $€ / \mathrm{MWh}$, given that the expected price of Player 1 for $55 \mathrm{MWh}$ is $99.81 € / \mathrm{MWh}$. Over its 100 executions, on average, the PSO method was able to obtain a profit of $5359.61 € / \mathrm{MWh}$, with a standard deviation of $314.31 € / \mathrm{MWh}$. This way, it is possible to confirm the high efficiency of the PSO approach to solve the problem of this case study scenario, being close to the optimal solution over its 100 executions. 


\section{Conclusions}

The development of a new DSS was motivated by the lack of opponent analysis in the pre-negotiation phase of bilateral contracts. By applying a game theory approach, the tool identifies the best action of all the possible actions that the supported player could take. However, the execution time of this approach greatly increases as the energy to trade and the number of opponents increases.

This paper proposed the use of PSO, a meta-heuristic algorithm, as an alternative approach to reduce execution time while keeping the results quality. The new approach was validated by the conducted case study as it provided optimal and near-optimal solutions in $4 \%$ of the time required by the first approach.

As future work, the proposed approach will consider the opponent's reputation. A multi-objective optimization approach will also be considered, including as additional optimization objective, the negotiation risk, based on the players' reputation.

\section{References}

1. L. Meeus, K. Purchala, and R. Belmans, "Development of the internal electricity market in europe," The Electricity Journal, vol. 18, no. 6, pp. 25 - 35, 2005.

2. E. Commission, "Renewable energy statistics." http://ec.europa.eu/eurostat/ statistics-explained/index.php/Renewable_energy_statistics, 2018. [Online; accessed 5-February-2018].

3. F. Lopes, M. Wooldridge, and A. Q. Novais, "Negotiation among autonomous computational agents: principles, analysis and challenges," Artificial Intelligence Review, vol. 29, no. 1, pp. 1-44, 2008.

4. T. Veselka et al., "Simulating the Behavior of Electricity Markets with an Agentbased Methodology: the Electric Market Complex Adaptive Systems (emcas) Model," 2002.

5. R. Lin et al., "GENIUS: An Integrated Environment for Supporting the Design of Generic Automated Negotiators," Computational Intelligence, vol. 30, no. 1, pp. $48-70,2014$.

6. F. Lopes, T. Rodrigues, and J. Sousa, "Negotiating Bilateral Contracts in a Multiagent Electricity Market: A Case Study," in 2012 23rd International Workshop on Database and Expert Systems Applications, pp. 326-330, 2012.

7. F. Silva et al., "Decision support system for the negotiation of bilateral contracts in electricity markets," in 8th International Symposium on Ambient Intelligence (ISAmI 2017), pp. 159-166, Springer International Publishing, 2017.

8. R. Eberhart and J. Kennedy, "A new optimizer using particle swarm theory," in Micro Machine and Human Science, 1995. MHS '95., Proceedings of the Sixth International Symposium on, pp. 39-43, Oct 1995.

9. T. Pinto, Z. Vale, I. Praça, E. J. S. Pires, and F. Lopes, "Decision support for energy contracts negotiation with game theory and adaptive learning," Energies, vol. 8, no. 9, pp. 9817-9842, 2015.

10. R. Faia, T. Pinto, and Z. Vale, "Dynamic fuzzy clustering method for decision support in electricity markets negotiation," ADCAIJ: Advances in Distributed Computing and Artificial Intelligence Journal, vol. 5, no. 1, 2016.

11. OMIE, "Market Results." http://www.omie.es/aplicaciones/datosftp/ datosftp.jsp, 2018. [Online; accessed 5-February-2018]. 